\title{
Cognitive or behavioural interventions (or both) to prevent or mitigate loneliness in adolescents, adults, and older adults (Protocol)
}

Eddy E, Heron P, McMillan D, Dawson S, Ekers D, Hickin N, Littlewood E, Shafran R, Meader N, Gilbody S

Eddy E, Heron P, McMillan D, Dawson S, Ekers D, Hickin N, Littlewood E, Shafran R, Meader N, Gilbody S.

Cognitive or behavioural interventions (or both) to prevent or mitigate loneliness in adolescents, adults, and older adults (Protocol).

Cochrane Database of Systematic Reviews 2020, Issue 11. Art. No.: CD013791.

DOI: 10.1002/14651858.CD013791.

www.cochranelibrary.com 
TABLE OF CONTENTS

HEADER 1

ABSTRACT

BACKGROUND

OBJECTIVES

METHODS

ACKNOWLEDGEMENTS

REFERENCES

APPENDICES

WHAT'S NEW

HISTORY

CONTRIBUTIONS OF AUTHORS

DECLARATIONS OF INTEREST

SOURCES OF SUPPORT 
[Intervention Protocol]

\section{Cognitive or behavioural interventions (or both) to prevent or mitigate loneliness in adolescents, adults, and older adults}

Elizabeth Eddy1a, Paul Heron¹b, Dean McMillan¹, Sarah Dawson²,3, David Ekers ${ }^{1,4}$, Nisha Hickin 5,6 , Elizabeth Littlewood ${ }^{1}$, Roz Shafran 7 , Nicholas Meader 3,8 , Simon Gilbody ${ }^{1}$

1Mental Health and Addiction Research Group, Department of Health Sciences, University of York, York, UK. 2Population Health Sciences, Bristol Medical School, University of Bristol, Bristol, UK. ${ }^{3}$ Cochrane Common Mental Disorders, University of York, York, UK. ${ }^{4}$ Lanchester Road Hospital, Tees, Esk and Wear Valleys NHS Foundation Trust, Durham, UK. 5 South West London and St George's Mental Health NHS Trust, London, UK. ${ }^{6}$ Great Ormond Street Institute of Child Health, University College London, London, UK. 7 University College London, London, UK. ${ }^{8}$ Centre for Reviews and Dissemination, University of York, York, UK

${ }^{a}$ These authors contributed equally to this work. ${ }^{b}$ These authors contributed equally to this work

Contact address: Paul Heron, paul.heron@york.ac.uk.

Editorial group: Cochrane Common Mental Disorders Group.

Publication status and date: New, published in Issue 11, 2020.

Citation: Eddy E, Heron P, McMillan D, Dawson S, Ekers D, Hickin N, Littlewood E, Shafran R, Meader N, Gilbody S. Cognitive or behavioural interventions (or both) to prevent or mitigate loneliness in adolescents, adults, and older adults (Protocol). Cochrane Database of Systematic Reviews 2020, Issue 11. Art. No.: CD013791. DOI: 10.1002/14651858.CD013791.

Copyright @ 2020 The Cochrane Collaboration. Published by John Wiley \& Sons, Ltd.

\section{A B S T R A C T}

\section{Objectives}

This is a protocol for a Cochrane Review (intervention). The objectives are as follows:

- To examine the effects of structured psychological interventions, based on cognitive behavioural therapy (CBT) techniques, compared to all comparators on loneliness in adolescents, adults, and older adults with diagnoses of common mental disorders, or at risk of loneliness.

- To examine the effects of structured psychological interventions, based on CBT techniques, compared to all comparators on depression severity, anxiety severity, social connectedness, or quality of life in adolescents, adults, and older adults, with diagnoses of common mental disorders, or at risk of loneliness. 


\section{B A C K G R O U N D}

\section{Description of the condition}

Evidence for the harm caused by loneliness is growing. Loneliness is subjective; it is an emotional response within a person, when a deficit exists between the desired and actual quantity and quality of social engagement and relationships (Perlman 1981; Victor 2005). Social isolation is objective; it is described as having 'minimal quality of structural (e.g. frequency of social contact) and functional (e.g. emotional support, or companionship) support' and can include low density social networks that are not maintained through frequent engagement (Hayanga 2020; Wang 2017). Loneliness and isolation are different than living alone; it is possible to experience loneliness or social isolation whilst living with others (Smith 2019).

Loneliness is found to peak in adolescence and in older people (Steptoe 2013; Victor 2012). In the UK, over a third of people over 50 years of age report experiencing loneliness (Thomas 2015). One in five US adults experience loneliness, and there is evidence this is increasing (Cacioppo 2015). Many other sections of the population are identified to be at a greater risk of loneliness such as people with low quality social relationships, people living alone, and recently bereaved adults (Cohen-Mansfield 2016).

Transient loneliness may be a healthy adaptive behavioural strategy (Cacioppo 2014; Mann 2017), but long-term loneliness is associated with psychological changes and poorer health outcomes (Hawkley 2010; Holwerda 2014; Petitte 2015; Rico-Uribe 2016). Loneliness is a significant public health issue (Gerst-Emerson 2015; Yan 2014), which is associated with mental health problems (Steptoe 2013), and is linked with increased severity of depression and anxiety (Lasgaard 2016; Meltzer 2013). The presence of loneliness is also a risk factor for poor treatment response, and poorer prognosis among people at risk of, or with established, depression and anxiety (Wang 2020). Loneliness is associated with poorer cognitive performance, faster cognitive decline, and increased negativity and depressed cognition, indicating that healthy social activity is likely to be integral to cognition in humans (Cacioppo 2009). There are associations between loneliness and increased risk of Alzheimer's disease and cognitive impairment (Boss 2015; Wilson 2007), eating disorders (Levine 2012), sleep problems (Smagula 2016), and suicidal ideation and suicide attempts (Mezuk 2014). Long-term data show that the severity of loneliness is a predictor for mortality (Steptoe 2013), and is equivalent to the health risks caused by obesity, smoking, and physical inactivity (Holt-Lunstad 2015).

\section{Description of the intervention}

The evidence on effective interventions to prevent or reduce loneliness is largely unclear. Reviews have identified four main intervention strategies to reduce loneliness: i. improving social skills; ii. enhancing social support; iii. increasing opportunities for social contact; and iv. modifying social cognitions (Masi 2011). The Masi 2011 meta-analysis found that the most effective intervention strategies of the four are cognitive interventions that seek to change cognition related to social situations.

A review by Mann 2017 summarised the evidence on cognitive approaches to tackle loneliness, and showed mixed results among psychological interventions. A trial comparing online cognitive behaviour therapy (CBT) plus motivational interviewing with online CBT and brief advice in adolescents with depression, found that both interventions reduced loneliness at 12 months (Richards 2016). A separate study found that cognitive re-framing did not reduce loneliness, but the intervention led to greater perceived control over reducing loneliness (Conoley 1985). A feasibility trial of a mindfulness intervention found reduced loneliness scores among Chinese students (Lindsay 2019). The Mann 2017 review highlights that cognitive changes from loneliness can potentially overlap with mental processes that contribute to mental health problems; it is hypothesised that changing a person's mental processes can lead to a change in social behaviour, and reduced loneliness over time (Mann 2017). Brief psychological therapies are advantageous, as they may be useful for people with chronic loneliness in the context of social isolation, or with non-clinical populations who are at risk of developing clinical depression (Gilbody 2017). A recent review of interventions for social isolation among people experiencing mental health problems found that the majority of studies had small sample sizes or did not sufficiently describe allocation concealment, missing data, and blinding procedures, meaning the evidence was not sufficient to make clinical recommendations ( $\mathrm{Ma}$ 2020). However, positive results on loneliness were reported in two out of six trials that examined interventions with a cognitive modification component and this led the authors to conclude that such interventions are 'promising'. Evidence for the effects of cognitive interventions on social isolation and other dimensions of social connectedness is also mixed; one trial of online behavioural activation versus specialised CBT for mothers with postpartum depression did not show positive effects on perceived social support (O'Mahen 2014), and a trial of behavioural activation for moderate depression among university students found a moderate, but not statistically significant effect on social support (Gawrysiak 2009).

This review will focus on trials of first, second, and third wave CBT interventions. This will include interventions directly targeting loneliness, or interventions for common mental health problems for which loneliness outcomes are reported. First wave CBT interventions are based on learning (behavioural) theory, and include classical conditioning and operant conditioning models. Interventions that use these approaches include exposure-based treatments for anxiety disorders, and behavioural treatments for depression (Abramowitz 2019). Exposure-based treatments involve coming into contact with a fear-provoking stimulus, and remaining in contact with it until anxiety reduces (extinction learning). Behavioural treatments for depression involve monitoring the link between mood and activity by scheduling activities to assess their impact on mood, and recording the results in a diary (Martell 2013).

Second wave CBT is based on a standard, Beckian account of emotional disorders (Beck 1979). This assumes that previous experiences lead to the development of a schema (core beliefs) that when activated, bias the way information is processed, which leads to emotional distress. Treatment techniques based on this approach include Socratic questioning of negative thoughts and behavioural experiments. In Socratic questioning, the therapist gently explores how accurate or helpful particularly negative thoughts are to the person. Outside of sessions, the person is encouraged to spot and challenge other negative thoughts in a similar way, often using a thought diary. Behavioural experiments involve doing something (a behaviour) to test the accuracy of a thought (an experiment, e.g. going to a gathering to test out the 
belief that 'no one will talk to me'). Second wave CBT typically incorporates techniques from first wave behavioural approaches, though these are often conceptualised from a cognitive, rather than a behavioural perspective (e.g. keeping a diary of moods, to test the belief that there is no variation in a person's low mood).

Third wave CBT approaches include a wide range of extensions to first and second wave CBT. These may be underpinned by cognitive theory (e.g. the meta-cognitive model) or behavioural theory (e.g. relational frame theory (Hayes 2004). Unlike second wave CBT, which focuses on the content of cognition, third wave CBT tends to place more emphasis on the processes and functions of how we relate to internal stimuli, such as our thoughts. Treatments typically classed as third wave CBT include acceptance and commitment therapy, dialectical behaviour therapy, meta-cognitive therapy, and mindfulness-based cognitive therapy. Treatment techniques used in a third wave approach vary substantially, depending on the particular type of treatment, although they often focus on practising a person's response to internal events (thoughts and feelings) in a new way (e.g. practising mindfulness, so that thoughts can be observed rather than getting caught up with them).

Cognitive behavioural therapy for loneliness targets the perceptual and cognitive biases that result in hypervigilance to negative social information (Cacioppo 2006; Cacioppo 2009). CBT helps people to look for disconfirming evidence to re-frame their perceptions of loneliness and self-efficacy, with the aim of changing avoidant behaviours, increasing social connections, and decreasing loneliness (Kall 2020). The key techniques include psychoeducation, social behaviour activation, exposure to social situations, the challenging of negative automatic thoughts, and behavioural experiments.

\section{How the intervention might work}

While there is substantial evidence that CBT treatments are effective, there is less consensus about how these treatments work generally, or specifically, to reduce loneliness. First wave behavioural explanations draw on classical conditioning models, such as extinction learning in exposure-based treatments. They also draw on operant conditioning principles, which argue that improvements in mental health outcomes occur when there is a reduction in behaviours that in the short-term are negatively reinforced, through reducing unpleasant internal states, such as anxiety, and establishing or re-establishing patterns of behaviour that in the long-term are linked to improvements in affective states. As applied to loneliness, these approaches would predict that encouraging people to engage in patterns of behaviours that establish or re-establish social interaction, even if this is difficult in the short-term, may reduce a person's sense of loneliness.

Second wave cognitive approaches suggest that cognitive biases that contribute to mental health problems could overlap with those in people experiencing loneliness (Mann 2017); for example, negative evaluations of other people are associated with both mental health problems and loneliness (Hawkley 2010). Therefore, psychological interventions may be able to address mental processes that are linked with loneliness, and support people to develop different thinking styles when thinking about themselves and their relationships. Mann 2017 hypothesise that these changes can result in changes to social behaviours that subsequently reduce loneliness.
Third wave approaches draw on a variety of both cognitive and behavioural theoretical explanations of emotional change that expand on, or revise, the first and second wave accounts. These have not as yet been extensively applied to understanding loneliness.

Finally, non-specific, or common factor models suggest that change in CBT and other psychological treatments rests on therapeutic practices that are common across all psychological treatments, such as developing a credible treatment rationale, agreement about the goals of therapy, and establishing an effective working relationship.

\section{Why it is important to do this review}

Large sections of the population have become socially isolated during the COVID-19 pandemic; either as a public health measure to prevent the transmission of COVID-19 in the community, or to protect those who are especially vulnerable. This is of particular concern for the millions of older adults and adolescents who are at greater risk of loneliness. Most existing loneliness research has focused on older adults but there has been less exploration of loneliness in people with, or at risk of developing, mental health problems, and strategies to tackle the problem. Whilst social isolation is likely to be a problem for many people, it is not inevitable that loneliness and mental health problems will follow, and there is a potential for psychological interventions to be used as public health measures.

Cultural changes mean that how people socialise is changing; the effects of this on loneliness are unknown. The UK National Health Service's (NHS) Five-year forward view for mental health report states the need to fulfil people's wishes for good quality relationships (Mental Health Task Force 2016). There has also been a UK initiative to understand the social determinants of mental health problems, and loneliness is becoming an emerging field of research following recent findings that highlight its potentially severe health impacts (Royal College of Psychiatrists 2010). There is an urgent need for evidence to address this problem.

To date, there is no well-developed evidence base supporting the effects of any intervention on loneliness, but a recent scoping review reported that psychological interventions have been the most extensively studied, and have the most robust evidence (Mann 2017). Despite this, evidence for the effectiveness of psychological interventions that can improve loneliness is mixed, and it is unclear what forms of therapeutic intervention and what factors, such as mode of delivery or setting, affect the success of such interventions. There is a clear need to review the evidence on this subject, but a Cochrane Review has not yet been conducted. Given the unclear evidence and a surge in subject interest, it is now timely to review the evidence, so that public health and clinical practice can be informed.

\section{OB JECTIVES}

- To examine the effects of structured psychological interventions, based on cognitive behavioural therapy (CBT) techniques, compared to all comparators on loneliness in adolescents, adults, and older adults with diagnoses of common mental disorders, or at risk of loneliness.

- To examine the effects of structured psychological interventions, based on CBT techniques, compared to all 
comparators on depression severity, anxiety severity, social connectedness, or quality of life in adolescents, adults, and older adults, with diagnoses of common mental disorders, or at risk of loneliness.

\section{METHODS}

\section{Criteria for considering studies for this review \\ Types of studies}

Randomised controlled trials (RCTs) will be eligible for inclusion. We decided to base the review on RCTs because of the robust nature of the trial design, which reduces bias arising from selection and allocation of groups. We will also include RCTs that use cluster allocation, cross-over elements, and trials with any time frame for outcome follow-up.

We will include studies regardless of their language of publication and date published. We will include studies regardless of their publication status, which will encompass unpublished and partially published studies, and those published in the grey literature.

We will exclude non-randomised studies, e.g. quasi-RCTs, as they allocate participants to intervention and control groups without strict randomisation; including these studies could increase the risk of allocation bias.

\section{Types of participants}

\section{Characteristics}

We will include studies with participants who are at least 10 years old. We will define adolescence in line with the World Health Organization (WHO) definition as those aged 10 to 19 years old (WHO 2014). We will exclude trials that include participants under the age of 10. If the age range straddles this cutoff, we will include the trial if we can establish that $>90 \%$ of the sample are older than 10 years of age. The rationale for selecting this age range is based on knowledge that loneliness is found to be most prevalent in adolescence and in older people (Victor 2012). We will not determine eligibility by any specific demographic features other than age.

\section{Setting}

We will include trials carried out in all settings (primary, secondary, or community).

\section{Diagnosis}

We will include participants with diagnoses of common mental health problems, such as anxiety, depression, obsessive compulsive disorder, phobias, panic disorders, and post-traumatic stress disorder. We will also include participants with no clinical diagnosis of common mental health problems, but who are deemed to be at a greater risk of loneliness or social isolation according to the existing literature. This includes participant groups, such as new mothers, recently bereaved adults, people living alone, people with low quality social relationships, and older adults who are socially isolated (Cohen-Mansfield 2016).

The diagnosis of a common mental health problem must be based on a structured or semi-structured clinical interview, conducted to internationally recognised standards (e.g. International Classification of Diseases, Diagnostic and Statistical Manual of
Mental Disorders), or on a score above a recognised threshold on a validated depression or anxiety severity measure (e.g. Patient Health Questionnaire-9 (PHQ-9; Kroenke 2001), Beck Depression Inventory (BDI; Beck 1961), Generalised Anxiety Disorder Assessment-7 (GAD-7; Spitzer 2006), or the Beck Anxiety Inventory (BAI; Beck 1988)). For loneliness, we will include interventions that focus on preventing loneliness, as well as reducing pre-existing loneliness (this could be defined using a recognised loneliness scale, such as the University of California, Los Angeles (UCLA) loneliness scale (Russell 1978 )). We will exclude studies that use a single item loneliness scale, or a modified scale taken from a larger loneliness scale, if the selected components do not show psychometric properties.

For studies that involve a subset of relevant participants, we will include the study if $90 \%$ of the participants meet our eligibility criteria. To ensure that this does not introduce review author's biases, we will conduct sensitivity analyses to assess the affect of the subset of data on the overall outcomes.

We suspect that there will be an insufficient number of studies to explore differences between studies with special circumstances of loneliness, such as incarceration or institutionalisation compared to those who are not. If there are sufficient studies, we will explore this through meta-regression.

\section{Comorbidities}

We will include trials in which participants have concurrent physical health problems, and those with a clinical diagnosis of mild to moderate dementia, or cognitive decline. We will exclude trials where participants have a clinical diagnosis of severe and enduring mental health problems, such as schizophrenia and bipolar disorder, severe cognitive decline, or a clinical diagnosis of a development disability, such as autism spectrum disorder.

\section{Types of interventions}

\section{Experimental Intervention}

Eligible interventions include first, second, or third wave cognitive behavioural therapies (CBT) seeking to improve or prevent loneliness, as well as other CBT interventions where the focus is on improving common mental health problems, but in which loneliness or a related construct is measured as an outcome.

We will also include trials that examine the effects of a full CBT intervention combined with other approaches; we will note the combination treatment in the interpretation of the findings.

\section{Format of psychological therapies}

The interventions can differ in terms of the mode of delivery (e.g. one-to-one, group, face-to-face, bibliotherapy, telephone, computerised, Internet, blended), the amount of support offered (e.g. pure self-help, minimal support, at least 20 one-hour sessions), and the level of training of the person providing the intervention (e.g. non-therapist technical support, limited training, fully qualified practitioner). We will include all combinations of mode, support level, and provider training level.

We will incorporate studies using interventions that are delivered at varying frequencies, intensity, and time scales. We will include studies with any length of follow-up. 
There is a risk that including all CBT interventions of varying format, delivery, and intensity may affect the intervention effect size. It will be important to consider the level of intensity of the therapy when reviewing the outcomes of the trials.

\section{Comparators}

All comparators are eligible for inclusion, including usual care, waiting-list control, or active comparators. Active comparators include: pharmacological interventions, non-CBT psychological interventions, and other CBT interventions (including dismantling or additive designs involving different combinations of CBT components in different treatment arms).

We have provided the details of how the different interventions and comparators will be grouped for analysis in the analysis section, including pre-planned main comparisons, subgroup analyses, and sensitivity analyses.

\section{Excluded interventions}

We will exclude all trials that do not use CBT (first, second, or third wave) as the form of intervention. We will not include interventions that contain only a single CBT component; for example, we would exclude a non-CBT intervention package that includes social skills training.

\section{Types of outcome measures}

Primary and secondary outcomes are outlined below.

\section{Primary outcomes}

- Loneliness, measured by a standard instrument that uses a validated continuous scale, such as the UCLA Loneliness Scale (Russell 1978 ) or the De Jong Gierveld Loneliness Scale (De Jong Gierveld 1985). We will accept the trial authors' original definition of loneliness.

\section{Secondary outcomes}

- Depression severity, measured by a standard instrument that uses a validated continuous scale, such as the PHQ-9 (Kroenke 2001), Beck Depression Inventory (Beck 1961), Hamilton Rating Scale for Depression (Hamilton 1960), MontgomeryAsberg Depression Rating Scale (Montgomery 1979), or other standardised depression scales.

- Anxiety severity, measured by a standard instrument that uses a validated continuous scale, such the Hamilton Anxiety Sale (Hamilton 1959), Beck Anxiety inventory (Beck 1988), the trait subscale of the Spielberger State-Trait Anxiety Inventory (Spielberger 1983), GAD-7 (Spitzer 2006), or other standardised anxiety scales.

- Quality of life, measured by a standard instrument that uses a validated continuous scale, such as Short Form-36 (Ware 1992), EuroQol (Brooks 1996), World Health Organization Quality of Life scales (WHOQOL Group 1998), or other validated measure of quality of life.

- Social connectedness (e.g. social support, social networks, and social isolation), measured by a standard instrument that uses a validated continuous scale, such as the Lubben Social Network Scale (Lubben 1988), or the Social Provision Scale (SPS; (Cutrona 1987)).

- Adverse effects, defined by individual studies. We will report the number and nature of each adverse effect for each study.
Reporting one or more of the secondary outcomes is not an inclusion criterion for the review. When a published report does not appear to report one of these outcomes, we will access the trial protocol and contact the trial authors to ascertain whether the outcomes were measured but not reported. We will include relevant trials that measured these outcomes, but did not report the data at all, or not in a usable format, as part of the narrative.

\section{Search methods for identification of studies}

\section{Electronic searches}

An information specialist with the Cochrane Common Mental Disorders (CCMD) Group will search the following bibliographic databases, using relevant subject headings, keywords, and search syntax appropriate to each resource.

- Cochrane Common Mental Disorders Controlled Trials Register (CCMDCTR; all available years; Appendix 1);

- Cochrane Central Register of Controlled Trials (CENTRAL; current issue, in the Cochrane Library);

- MEDLINE Ovid (1946 onwards);

- Embase Ovid (1974 onwards);

- PsycINFO Ovid (all years; Appendix 2);

- Web of Science Social Science Citation Index (SSCl; all years);

- Social Care Online (www.scie-socialcareonline.org.uk; all years).

We will search ClinicalTrials.gov and the World Health Organization (WHO) International Clinical Trials Registry Platform (ICTRP; all years) to identify ongoing or unpublished trials.

We will not apply any restriction on date, language, or publication status to the searches.

\section{Searching other resources}

\section{Grey literature}

We will search the following sources of grey literature (primarily for dissertations and theses):

- Open Grey (www.opengrey.eu/);

- ProQuest Dissertations \& Theses Global (www.proquest.com/ products-services/pqdtglobal.html);

- DART-Europe E-theses Portal (www.dart-europe.eu/);

- EThOS - the British Libraries e-theses online service (ethos.bl.uk/);

- Networked Digital Library of Theses and Dissertations (NDLTD; search.ndltd.org);

- Open Acces Theses and Dissertations (oatd.org).

\section{Reference lists}

We will check the reference lists of all relevant study reports and reviews retrieved by the searches. We will also conduct a forward citation search on the Web of Science and Google Scholar, to identify additional research.

\section{Personal communication}

We will contact trial authors and subject experts for information on unpublished or ongoing studies, or to request additional data. 


\section{Data collection and analysis}

\section{Selection of studies}

Two review authors (PH, EE) will independently screen titles and abstracts of all the potential studies we identify as a result of the search, and code them as 'retrieve' (eligible or potentially eligible or unclear) or 'do not retrieve', using Covidence software (Covidence). If there are any disagreements, a third review author will be asked to arbitrate (DM). We will retrieve the full-text study reports or publication of those coded 'retrieve'; two review authors ( $\mathrm{PH}, \mathrm{EE})$ will independently screen the full-text, identify studies for inclusion, and identify and record reasons for exclusion of the ineligible studies. We will resolve any disagreement through discussion, or if required, we will consult a third person (DM). We will identify and exclude duplicates and collate multiple reports of the same study, so that each study, rather than each report, is the unit of interest in the review. We will record the selection process in sufficient detail to complete a PRISMA flow diagram and 'Characteristics of excluded studies' table (Liberati 2009). When the full text is not available we will first contact the trial author. If we receive no response, we will contact the British Library to gain access, or purchase the study via the University of York where possible.

\section{Data extraction and management}

We will use a data collection form for study characteristics and outcome data, which has been piloted on at least one study in the review. Two review authors (PH, EE) will independently extract study characteristics from included studies. We will extract the following study characteristics into Covidence software (Covidence).

- Methods: study design, total duration of study, number of study centres and location, study setting, and date of study

- Participants: total randomised, number lost to follow-up or withdrawn, number analysed, mean age, age range, gender, severity of condition, medications taken, diagnostic criteria, inclusion criteria, and exclusion criteria

- Interventions: intervention, comparison, mode of delivery, frequency, length of sessions, treatment duration, level of support provided by treatment providers, level of training of treatment providers

- Outcomes: we will collect outcomes of loneliness, depression severity, anxiety severity, quality of life, social support, social networks and social isolation, and report the time points

- Notes: funding for trial, notable conflicts of interest of trial authors, and adverse events

Two review authors (PH, EE) will independently extract outcome data from included studies. We will resolve disagreements by consensus, or by involving a third review author (DM). One review author (EE) will import data from Covidence into the Review Manager 5 file (Covidence; Review Manager 2014). We will double check that data are entered correctly by comparing the data presented in the Review Manager 5 file with the data extraction form.

\section{Main comparisons}

- CBT versus the control condition

- CBT versus other active psychological treatments

\section{- CBT versus active pharmacological treatment}

\section{Assessment of risk of bias in included studies}

Two review authors ( $\mathrm{PH}, \mathrm{EE})$ will independently assess risk of bias for each study using version 2 of the Cochrane 'Risk of bias' (RoB 2) tool (Sterne 2019), outlined in the Cochrane Handbook for Systematic Reviews of Interventions (Higgins 2019b). We will assess the risk of bias of the results for a specific outcome in each trial according to the following domains:

1. Bias arising from the randomisation process; randomisation and allocation

2. Bias due to deviations from intended interventions (assignment to intervention);

3. Bias due to missing outcome data;

4. Bias in the measurement of the outcome;

5. Bias in the selection of the reported result.

We will assess the risk of bias for the outcomes of the included trials that will be included in our 'Summary of findings' table. We will quantify the effect of assignment to the interventions at baseline, regardless of whether the interventions are received as intended (the intention-to-treat effect; (Higgins 2019b)).

We will use the signalling questions in the 'RoB 2 ' tool, and rate each domain as low risk of bias, some concerns, or high risk of bias. We will summarise the risk of bias judgements across different studies for each of the domains listed for each outcome. The overall risk of bias for the result is the least favourable assessment across the domains of bias. When considering treatment effects, we will take into account the risk of bias for the studies that contribute to that outcome.

Cross-over and cluster-randomised control trials bias (including the five RoB 2 categories mentioned previously) will be analysed using a more suited method that focusses on common biases produced from these trial formats. We will analyse cluster-randomised trials for bias that arises from the randomisation process, baseline imbalances, missing outcome data, measurement of the outcome, selection of the reported result, and loss of cluster (Higgins 2020a). We will analyse cross-over trials for bias that arises from the randomisation process, carry-over effects, missing outcome data, measurement, and selection of the reported results (Higgins 2020a).

Where inadequate details are provided in the original report, we will contact corresponding trial authors to provide clarification. Disagreements will be resolved following discussions with DM.

\section{Measures of treatment effect}

We will manipulate data presented as a scale so that there is a consistent direction of effect. We will narratively describe skewed data, reported as medians and interquartile ranges.

\section{Dichotomous dato}

We will analyse dichotomous outcomes by calculating a pooled risk ratio (RR) and 95\% confidence intervals (95\% Cls).

A score over one suggests a benefit. We will analyse dichotomous data using an odds ratio measurement. We will then convert these odds ratios into risk ratios to allow easier interpretation, using 
the formula described in the Cochrane Handbook for Systematic Reviews of Interventions (Schünemann 2020b). For measures that use a cutoff score to determine dichotomous data, we will report the cutoff score used in each study, and address differences in sensitivity in the narrative synthesis.

We will also calculate the risk difference (RD), which in turn, can be used to calculate the number needed to treat for an additional beneficial outcome (NNTB), which is another way to analyse the outcome effect. The NNTB is defined as the expected number of people needed to receive the intervention compared to the comparator group for one additional person to either avoid or achieve an outcome in given time scale (Schünemann 2020a)

\section{Continuous dato}

If all the studies for a particular outcome of interest use the same standardised measure, we will report a mean difference (MD) with $95 \% \mathrm{Cl}$.

If the trials use different instrument to measure the outcome, we will calculate a standardised mean difference (SMD) with $95 \% \mathrm{Cl}$. The standardised mean difference measures the size of the intervention effect of each study compared to the betweenparticipant variability in outcome measurements recorded in each individual study (Schünemann 2020b). To allow for easier interpretation and analysis of the results, we will convert the SMD into the specific clinical measures used in the studies (such as the UCLA Loneliness Scale (Russell 1978 ), or the De Jong Gierveld Loneliness Scale (De Jong Gierveld 1985), as this is a more familiar unit of measurement. This is done by multiplying the SMD by an estimate of the standard deviation (SD) associated with the outcome measure instrument, according to the Handbook (Schünemann 2020b).

\section{Management of time points}

We plan to categorise the post-intervention outcomes into shortterm outcomes ( $<6$ months, including end of treatment time points), medium-term (6 to 12 months), and long-term outcomes ( $\geq 12$ months). If a study reports follow-up outcomes at more than one time point within one of these time frames, we will select the outcome reported at the latest point within the time frame.

\section{Hierarchy of outcomes}

We will separately analyse symptom severity (e.g. depression or anxiety severity typically using continuous measures) and diagnostic outcomes (typically assessed using dichotomous outcomes), and will consider symptom severity as having priority over diagnostic outcomes. If a trial reports the use of more than one instrument assessing for each outcome, we will prioritise in the following order: clinician-rated scale, informant-rated scale, and self-rated scale. If multiple outcome measures are used of the same type (e.g. two self-report measures), we will choose the outcome measure that is most frequently used across studies. If multiple outcome measures of the same type have equivalent availability across studies, we will choose the one with the strongest psychometric evidence in previous research.

\section{Unit of analysis issues}

\section{Cross-over trials}

We will include trials that use a cross-over element, using only data extracted from the first trial period. Our decision to only include the first phase was based on the risk of 'carry over' effects (when the effects of the previous intervention continue to have an effect in the subsequent trial phase) and 'period' effects (changing of the underlying condition systematically or background factors alter which can often arise from cross-over trials; (Higgins 2020a)).

\section{Cluster-randomised trials}

We will include trials that use cluster-randomisation. In order to include cluster-randomised trials correctly, we will follow the advice given in the Cochrane Handbook for Systematic Reviews of Interventions (Higgins 2020a).

\section{Trials with multiple treatment groups}

Studies that include multiple-arms run the risk of introducing a unit-of-analysis error if the data are not managed appropriately. To combat this issue, we will take the following approach:

- If trials include multiple relevant treatment groups, we will combine relevant intervention groups and useful comparator groups (into active or control) in a pair-wise comparison;

- If trials include multiple active comparator groups, we will combine these to compare one active group with the intervention group;

- If trials include multiple usual care, placebo, or waiting-list comparator groups, we will combine these to compare one nonactive group with the intervention group;

- If trials include multiple relevant interventions with a single control group, we will divide the control group in pair-wise comparisons.

\section{Dealing with missing data}

We will contact investigators or study sponsors in order to verify key study characteristics and obtain missing numerical outcome data when needed, and when possible (e.g. when a study is identified as abstract only).

When standard deviations (SDs) are missing, we will attempt to obtain these data by contacting trial authors. When SDs are not available from trial authors, we will calculate them from $P$ values, $\mathrm{t}$-values, Cls, or standard errors, if these are reported in the articles (Deeks 1997).

If a vast majority of SDs are available, and only a minority of SDs are unavailable or unobtainable, we will use the method devised by Furukawa and colleagues to impute SDs and calculate percentage responders (da Costa 2012; Furukawa 2005, Furukawa 2006). If we use this method, we will interpret data with caution, and will take into account the degree of observed heterogeneity. We will also undertake a sensitivity analysis to examine the effect of the decision to use imputed data.

If additional figures are not available or obtainable, and it is not deemed appropriate to use the Furukawa method as described above, we will not include the trial data in the comparison of interest. 
Where there are missing data in included studies, we will include available case data (i.e. where data from only participants with a final assessment were included), or where trial authors imputed missing data, using techniques, such as multiple imputation, mixed model regression, or last observation carried forward (LOCF).

\section{Assessment of heterogeneity}

We will inspect forest plots visually to consider the direction and magnitude of effects and the degree of overlap between confidence intervals. We will use the $\mathrm{Chi}^{2}$ test to assess statistical heterogeneity, and this will provide evidence of variation in effect estimates beyond that of chance. The $\mathrm{Chi}^{2}$ test has low power to assess heterogeneity when a low number of participants or trial are included, so we will conservatively set the $\mathrm{P}$ value at 0.1 (Deeks 2020). We will also quantify heterogeneity using the $I^{2}$ statistic, giving us values that calculate the percentage of variability attributed to heterogeneity rather than to chance (Deeks 2020). We will consider $\mathrm{I}^{2}$ values in the range of $50 \%$ to $90 \%$ to represent substantial statistical heterogeneity, and we will explore such cases further.

The magnitude and direction of treatment effects will affect the importance of the observed $I^{2}$ statistic. The forest plots will provide an estimate of the $\mathrm{Tau}^{2}$ between-trial variance in a random-effects meta-analysis (Deeks 2020). We will use the $\mathrm{Tau}^{2}$ estimate to determine an approximate range of intervention effects for the primary outcome; this will indicate the true spread of the effects of the intervention (Deeks 2020).

\section{Assessment of reporting biases}

To minimise the risk of reporting bias, we will conduct extensive searches to include a range of English and non-English published trials, unpublished trials, clinical study reports, and trial registries data in the review. By doing this, it will minimise the risk of bias due to missing results.

We will also attempt to identify outcome reporting bias in trials by recording all relevant trial outcomes, planned and reported, and noting where outcomes are missing. If we find evidence of missing outcomes, we will attempt to obtain any available data by directly contacting the trial authors.

If we are able to pool more than 10 trials, we will create and examine a funnel plot to explore possible small study biases for the primary outcomes. We will construct a funnel plot of effect size versus inverse variance to test for publication bias. An asymmetrical funnel plot is strongly indicative of publication bias, and means that the results of the meta-analysis should be interpreted with caution (Egger 1997).

\section{Data synthesis}

We will initially group studies and report by: time frame (short-, medium-, and long-term), comparator conditions (active versus control, and active versus active), and participant age category (adolescent, adult, or older adult). We will use the WHO's definitions of adolescence (aged 10 to 19 years; (WHO 2014)), and older adults (aged over 60 years; (WHO 2001)). We will consider adults as aged 18 years and over.

We will undertake meta-analyses only where this is meaningful, i.e. if the treatments, participants, and the underlying clinical question are similar enough for pooling to make sense.
We will use a random-effects model in the analyses, as we predict a high rate of heterogeneity between the selected studies. A random-effects meta-analysis model suggests that the effects being estimated in different studies follow some sort of distribution (Deeks 2020).

\section{Narrative synthesis}

If we carry out a narrative synthesis, we will develop a theory of how the intervention might work, according to the included studies. Then, we will conduct a preliminary synthesis by systematically and thoroughly assessing each study to extract relevant information, in order to evaluate the similarities and differences between them. With this information, we will look closely at the relationships of the data within and between studies. Finally, we will assess how robust our synthesis has been, by looking at the methods used to complete the synthesis, and the quality of the data produced.

\section{Subgroup analysis and investigation of heterogeneity}

We plan to carry out the following subgroup analyses, if there are sufficient studies to do so:

- Delivery of the therapy: we will categorise the interventions depending on their mode of delivery. This will allow us to compare the difference between interventions delivered faceto-face versus those delivered via remote means (telephone, web based)

- Therapy support: we will categorise therapies depending on the level of support provided, such as supported versus unsupported. We predict that there will be a positive association between the level of support provided and the effectiveness of the therapy.

- Type of psychological therapy: we will categorise the form of psychological therapy, where possible, into generic psychological therapies versus therapy that focuses on targeting loneliness. We anticipate that interventions that directly target loneliness could have a greater positive effect on the outcomes.

- Diagnostic equivalence: we will categorise the diagnostic method, based on the tool used, for studies that include a measure of mental health, for example studies which use a screening tool (e.g. PHQ9) versus a diagnostic method (e.g. meeting the DSM or ICD criteria for a mental disorder). This will allow us to assess if effectiveness estimates differ in studies that include participants with a common mental disorder, based on a rating scale or a diagnostic tool.

- Type of therapy: we will categorise interventions based on whether first, second, or third wave techniques were used to compare differences between these types.

We will explore the relationship between effect size and methodological features identified in the 'Risk of bias' assessment using a meta-regression. If there are not sufficient studies to conduct a statistical analysis, we will address this in the narrative synthesis.

We will use the formal test for subgroup differences in Review Manager 5, and base our interpretation on this (Review Manager 2014). 


\section{Sensitivity analysis}

We plan to carry out the following sensitivity analyses, to test whether key methodological factors or decisions affected the main result:

- Trial quality: we will exclude trials that are at greater risk of bias (lower quality). We will define quality using domains of the 'Risk of bias 2' tool, including allocation concealment and attrition rate. If there are differences in risk of bias between domains, then the rating indicating the higher risk of bias will be used.

- Multi-component or complex therapy: we will exclude multicomponent or complex therapies. This will show the effect of the additional components in the therapies, compared with generic CBT alone.

\section{Reaching conclusions}

We will base our conclusions only on findings from the quantitative or narrative synthesis of studies included in this review.We will avoid making recommendations for practice; our implications for research will suggest priorities for future research, and outline the remaining uncertainties in the area.

\section{Summary of findings and assessment of the certainty of the evidence}

We will create a 'Summary of findings' table using the following outcomes:

- Loneliness

- Depression severity

- Anxiety severity

- Quality of life

- Social connectedness, i.e. social support, social networks, and social isolation

- Adverse events

We will use the five GRADE considerations (study limitations, consistency of effect, imprecision, indirectness, and publication bias) to assess the quality of the body of evidence as it relates to the studies that contribute data to the meta-analyses for the prespecified outcomes. We will use methods and recommendations described in Chapter 14 of the Cochrane Handbook for Systematic Reviews of Interventions (Schünemann 2020a), and GRADEpro software (GRADEpro GDT). We will create a separate 'Summary of findings' table for each comparison (CBT versus the control condition, and CBT versus other active psychological treatments). We will justify all decisions to downgrade the quality of the evidence using footnotes, and we will make comments to aid the reader's understanding of the review, where necessary. We will downgrade by one level for each GRADE factor, up to a maximum of three levels for all factors. If there are very severe problems for a single factor, we will downgrade by two levels.

Examples of each factor for which we would downgrade the level of the evidence:
- study limitations: selective outcome reporting, or recruitment bias in cluster-randomised trials

- consistency of effect: unexplained heterogeneity of results

- imprecision: the presence of wide confidence intervals

- indirectness: time differences in measurement of outcomes

- publication bias: over-estimation of underlying beneficial effects due to publication selection bias

Two review authors (PH, EE), working independently, will make judgements about the quality of the evidence, with disagreements resolved by discussion, or involving a third review author (DM). Judgements will be justified, documented, and incorporated into the reporting of results for each outcome. The 'Summary of findings' table will be completed before writing the discussion, conclusions, and abstract, to highlight any potential influence the study quality had on the reported outcomes.

We will use these domains to rate the overall quality of evidence for the outcomes according to the following categories:

- High quality: further research is very unlikely to change our confidence in the estimate of effect:

- Moderate quality: further research is likely to have an important impact on our confidence in the estimate of effect, and may change the estimate;

- Low quality: further research is very likely to have an important impact on our confidence in the estimate of effect, and is likely to change the estimate;

- Very low quality: we are very uncertain about the estimate.

We will report other key information in the 'Summary of findings' table, such as study sample size and data collection time frame.

\section{ACKNOWLEDGEMENTS}

We are grateful for the advice and support received from the editorial team of the Cochrane Common Mental Disorders (CCMD) Group.

The review authors and the CCMD Editorial Team, are grateful to the peer reviewers for their time and comments including: Helen Brooks, Kristin Osika, Eiluned Pearce, Anil Raj, Akilesh Ramasamy, Lindsay Robertson and Eleonora Uphoff. They would also like to thank copy editor, Victoria Pennick.

SG, PH, DMcM were funded by the National Institute for Health Research Collaboration for Leadership in Applied Health Research and Care, Yorkshire and Humber (NIHR CLAHRC YH)

Cochrane Group funding acknowledgement: The National Institute for Health Research (NIHR) is the largest single funder of the Cochrane Common Mental Disorders Group.

Disclaimer: The views and opinions expressed herein are those of the review authors and do not necessarily reflect those of the NIHR, National Health Service (NHS), or the Department of Health and Social Care. 


\section{R E F E R E N C E S}

\section{Additional references}

\section{Abramowitz 2019}

Abramowitz JS, Deacon BJ, Whiteside SP. Exposure Therapy for Anxiety: Principles and Practice. 2nd edition. New York: Guilford Publications, 2019.

\section{Beck 1961}

Beck AT, Ward C, Mendelson M, Mock J, Erbaugh J. An inventory for measuring depression. Archives of General Psychiatry 1961;4(6):561-71.

\section{Beck 1979}

Beck AT, Rush AJ, Shaw BF, Emery G. Cognitive Therapy of Depression. 1st edition. New York: Guilford Press, 1979.

\section{Beck 1988}

Beck AT, Epstein N, Brown G, Steer RA. An inventory for measuring clinical anxiety: psychometric properties. Journal of Consulting and Clinical Psychology 1988;56(6):893-7.

\section{Boss 2015}

Boss L, Kang DH, Branson S. Loneliness and cognitive function in the older adult: a systematic review. International Psychogeriatrics 2015;27(4):541-53.

\section{Brooks 1996}

Brooks R. EuroQol: the current state of play. Health Policy 1996;37(1):53-72.

\section{Cacioppo 2006}

Cacioppo JT, Hughes ME, Waite LJ, Hawkley LC, Thisted RA. Loneliness as a specific risk factor for depressive symptoms: cross-sectional and longitudinal analyses. Psychology and Aging 2006;21(1):140-51. [DOI: 10.1037/0882-7974.21.1.140]

\section{Cacioppo 2009}

Cacioppo JT, Hawkley LC. Perceived social isolation and cognition. Trends in Cognitive Sciences 2009;13(10):447-54.

\section{Cacioppo 2014}

Cacioppo JT, Cacioppo S, Boomsma DI. Evolutionary mechanisms for loneliness. Cognition and Emotion 2014;28(1):3-21.

\section{Cacioppo 2015}

Cacioppo S, Grippo AJ, London S, Goossens L, Cacioppo JT. Loneliness: clinical import and interventions. Perspectives on Psychological Science 2015;10(2):238-49.

\section{Cohen-Mansfield 2016}

Cohen-Mansfield J, Hazan H, Lerman Y, Shalom V. Correlates and predictors of loneliness in older-adults: a review of quantitative results informed by qualitative insights. International Psychogeriatrics 2016;28(4):557-76. [DOI: 10.1017/ S1041610215001532]

\section{Conoley 1985}

Conoley CW, Garber RA. Effects of reframing and self-control directives on loneliness, depression, and controllability. Journal of Counseling Psychology 1985;32(1):139-42.

\section{Covidence [Computer program]}

Veritas Health Innovation Covidence. Version accessed 27 October 2020. Melbourne, Australia: Veritas Health Innovation. Available at covidence.org.

\section{Cutrona 1987}

Cutrona CE, Russell DW. The provisions of social relationships and adaptation to stress. In: Jones WH, Perlman D, editors(s). Advances in Personal Relationships. 1st edition. Stamford, CT: JAI Press, 1987:37-67.

\section{da Costa 2012}

da Costa Bruno R, Rutjes AWS, Johnston BC, Reichenbach S, Nüesch E, Tonia T, et al. Methods to convert continuous outcomes into odds ratios of treatment response and numbers needed to treat: meta-epidemiological study. International Journal of Epidemiology 2012;41(5):1445-59.

\section{Deeks 1997}

Deeks J. Are you sure that's a standard deviation? (part 1). Cochrane News 1997;10:11-2.

\section{Deeks 2020}

Deeks JJ, Higgins JPT, Altman DG, editor(s). Chapter 10: Analysing data and undertaking meta-analyses. In: Higgins JPT, Thomas J, Chandler J, Cumpston M, Li T, Page MJ, et al, editor(s). Cochrane Handbook for Systematic Reviews of Interventions version 6.1 (updated September 2020). Cochrane, 2020. Available from www.training.cochrane.org/handbook.

\section{De Jong Gierveld 1985}

De Jong-Gierveld J, Kamphuis F. The development of a Raschtype loneliness scale. Applied Psychological Measurement 1985;9(3):289-99.

\section{Egger 1997}

Egger M, Davey Smith G, Schneider M, Minder C. Bias in meta-analysis detected by a simple, graphical test. $B M J$ 1997;315:629-34.

\section{Furukawa 2005}

Furukawa TA, Cipriani A, Barbui C, Brambilla P, Watanabe N. Imputing response rates from means and standard deviations in meta-analyses. International Clinical Psychopharmacology 2005;20(1):49-52.

\section{Furukawa 2006}

Furukawa BA, Barbui C, Cipriani A, Brambilla P, Watanabe N. Imputing missing standard deviations in meta-analyses can provide accurate results. Journal of Clinical Epidemiology 2006;59(1):7-10. 


\section{Gawrysiak 2009}

Gawrysiak M, Nicholas C, Hopko DR. Behavioral activation for moderately depressed university students: randomized controlled trial. Journal of Counselling Psychology 2009;56(3):468-75.

\section{Gerst-Emerson 2015}

Gerst-Emerson K, Jayawardhana J. Loneliness as a public health issue: the impact of loneliness on health care utilization among older adults. American Journal of Public Health 2015;105(5):1013-9.

\section{Gilbody 2017}

Gilbody SM, Lewis HJ, Adamson JA, Atherton K, Bailey D, Birtwistle J, et al. Effect of collaborative care vs usual care on depressive symptoms among older adults with sub-threshold depression. Journal of the American Medical Association 2017;317(7):728-37.

\section{GRADEpro GDT [Computer program]}

McMaster University (developed by Evidence Prime) GRADEpro GDT. Version accessed prior to 27 October 2020. Hamilton (ON): McMaster University (developed by Evidence Prime). Available at gradepro.org.

\section{Hamilton 1959}

Hamilton M. The assessment of anxiety states by rating. British Journal of Medical Psychology 1959;32(1):50-5.

\section{Hamilton 1960}

Hamilton M. A rating scale for depression. Journal of Neurology, Neurosurgery and Psychiatry 1960;23(1):56.

\section{Hawkley 2010}

Hawkley LC, Cacioppo JT. Loneliness matters: a theoretical and empirical review of consequences and mechanisms. Annals of Behavioral Medicine 2010;40(2):218-27.

\section{Hayanga 2020}

Hayanga B, Kneale D, Phoenix A. Understanding the friendship networks of older Black and minority ethnic people living in the United Kingdom. Ageing and Society. Cambridge University Press 2020:1-20. [DOI: 10.1017/s0144686x19001624]

\section{Hayes 2004}

Hayes SC, Follette VM, Lineham M. Mindfulness and Acceptance: Expanding the Cognitive-Behavioural Tradition. 1st edition. New York: Guilford Press, 2004.

\section{Higgins 2019b}

Higgins JP, Savović J, Page MJ, Elbers RG, Sterne JA, editor(s). Chapter 8: Assessing risk of bias in a randomized trial. In: Higgins JP, Thomas J, Chandler J, Cumpston MS, Li T, Page MJ, et al, editor(s), Cochrane Handbook for Systematic Reviews of Interventions version 6.0 (updated October 2019). The Cochrane Collaboration, 2019. Available from www.training.cochrane.org/ handbook.

\section{Higgins 2020a}

Higgins JPT, Eldridge S, Li T, editor(s). Chapter 23: Including variants on randomized trials. In: Higgins JPT, Thomas J,
Chandler J, Cumpston M, Li T, Page MJ, et al, editor(s). Cochrane Handbook for Systematic Reviews of Interventions version 6.1 (updated September 2020). Cochrane, 2020. Available from www.training.cochrane.org/handbook.

\section{Holt-Lunstad 2015}

Holt-Lunstad J, Smith TB, Baker M, Harris T, Stephenson D. Loneliness and social isolation as risk factors for mortality: a meta-analytic review. Perspectives on Psychological Science 2015;10(2):227-37.

\section{Holwerda 2014}

Holwerda Tj, Deeg D, Beekman A, van Tilburg T, Stek M, Jonker C, et al. Feelings of loneliness, but not social isolation, predict dementia onset: results from the Amsterdam Study of the Elderly (AMSTEL). Journal of Neurology, Neurosurgery, and Psychiatry 2014;85(2):135-42.

\section{Kall 2020}

Kall A, Jaghol S, Hesser H, Andersson F, Mahaldi A, Norkvist BT, et al. Internet-based cognitive behavior therapy for loneliness: a pilot randomized controlled trial. Behavior Therapy 2020;51(1):54-68. [DOI: 10.1016/j.beth.2019.05.001]

\section{Kroenke 2001}

Kroenke K, Spitzer RL, Williams JB. The PHQ-9: validity of a brief depression severity measure. Journal of General Internal Medicine 2001;16(9):606-13.

\section{Lasgaard 2016}

Lasgaard M, Friis K, Shevlin M. "Where are all the lonely people?" A population-based study of high-risk groups across the life span. Social Psychiatry and Psychiatric Epidemiology 2016;51(10):1373-84.

\section{Levine 2012}

Levine MP. Loneliness and eating disorders. Journal of Psychology 2012;146(1):243-57.

\section{Liberati 2009}

Liberati A, Altman DG, Tetzlaff J, Mulrow C, Gøtzsche PC, Ioannidis JP, et al. The PRISMA statement for reporting systematic reviews and meta-analyses of studies that evaluate health care interventions: explanation and elaboration. PLOS Medicine 2009;6(7):e1000100.

\section{Lindsay 2019}

Lindsay EK, Young S, Brown KW, Smyth JM, Creswell D. Mindfulness training reduces loneliness and increases social contact in a randomized controlled trial. Proceedings of the National Academy of Sciences 2019;116(9):3488.

\section{Lubben 1988}

Lubben JE. Assessing social networks among elderly populations. Family \& Community Health 1988;11(3):42-52.

\section{Ma 2020}

Ma R, Mann F, Wang J, Lloyd-Evans B, Terhune J, Al-Shihabi A, et al. The effectiveness of interventions for reducing subjective and objective social isolation among people with mental health problems: a systematic review. Social Psychiatry and 
Psychiatric Epidemiology 2020;55(7):839-76. [DOI: 10.1007/ s00127-019-01800-z]

\section{Mann 2017}

Mann F, Bone JK, Lloyd-Evans B, Frerichs J, Pinfold V, Ma R, et al. A life less lonely: the state of the art in interventions to reduce loneliness in people with mental health problems. Social Psychiatry and Psychiatric Epidemiology 2017;52(6):627-38.

\section{Martell 2013}

Martell CR, Dimidjian S, Herman-Dunn R. Behavioural Activation for Depression: A Clinician's Guide. 1st edition. New York: Guilford Press, 2013.

\section{Masi 2011}

Masi CM, Hsi-Yuan C, Hawkley LC, Cacioppo JT. A meta-analysis of interventions to reduce loneliness. Personality and Social Psychology Review 2011;15(3):219-66.

\section{Meltzer 2013}

Meltzer H, Bebbington P, Dennis MS, Jenkins R, McManus S, Brugha TS. Feelings of loneliness among adults with mental disorder. Social Psychiatry and Psychiatric Epidemiology 2013;48(1):5-13.

\section{Mental Health Task Force 2016}

Mental Health Task Force, The NHS Confederation. The five year forward view for mental health. www.england.nhs.uk/ wp-content/uploads/2016/02/Mental-Health-Taskforce-FYFVfinal.pdf (accessed prior to 27 October 2020).

\section{Mezuk 2014}

Mezuk B, Rock A, Lohman MC, Choi M. Suicide risk in long-term care facilities: a systematic review. International Journal of Geriatric Psychiatry 2014;29(12):1198-211.

\section{Montgomery 1979}

Montgomery SA, Asberg M. A new depression scale designed to be sensitive to change. British Journal of Psychiatry 1979;134(4):382-9.

\section{O'Mahen 2014}

O'Mahen HA, Richards DA, Woodford J, Wilkinson E, McGinley J, Taylor RS, et al. Netmums: a phase II randomized controlled trial of a guided Internet behavioural activation treatment for postpartum depression. Psychological Medicine 2014;44(8):1675-89.

\section{Perlman 1981}

Perlman D, Peplau LA. Toward a social psychology of loneliness. In: Gilmour R, Duck S, editors(s). Personal Relationships. 3: Relationships in Disorder. London: Academic Press, 1981:31-56.

\section{Petitte 2015}

Petitte T, Mallow J, Barnes E, Petrone A, Barr T, Theeke L. A systematic review of loneliness and common chronic physical conditions in adults. The Open Psychology Journal 2015;8(1 Suppl 2):113-2.

\section{Review Manager 2014 [Computer program]}

The Cochrane Collaboration Review Manager 5 (RevMan 5). Version 5.4. Copenhagen: The Nordic Cochrane Centre: The Cochrane Collaboration, 2014.

\section{Richards 2016}

Richards K, Marko-Holguin M, Fogel J, Anker L, Ronayne J, Van Voorhees BW. Randomized clinical trial of an internet-based intervention to prevent adolescent depression in a primary care setting (catch-it): 2.5-year outcomes. Journal of Evidence-based Psychotherapies 2016;16(2):113-34.

\section{Rico-Uribe 2016}

Rico-Uribe LA, Caballero FF, Olaya Be, Tobiasz-Adamczyk B, Koskinen S, Leonardi M, et al. Loneliness, social networks, and health: a cross-sectional study in three countries. PloS one 2016;11(1):e0145264.

\section{Royal College of Psychiatrists 2010}

Royal College of Psychiatrists. No health without public mental health: the case for action. Position Statement PS4/2010. www.rcpsych.ac.uk/pdf/PS04_2010.pdf (accessed prior to 27 October 2020).

\section{Russell 1978}

Russell D, Peplau LA, Ferguson ML. Developing a measure of loneliness. Journal of Personality Assessment 1978;42(3):290-4.

\section{Schünemann 2020a}

Schünemann HJ, Higgins JP, Vist GE, Glasziou P, Akl EA, Skoetz N, et al, on behalf of the Cochrane GRADEing Methods Group. Chapter 14: Completing 'Summary of findings' tables and grading the certainty of the evidence. In: Higgins JP, Thomas J, Chandler J, Cumpston MS, Li T, Page MJ, et al, editor(s), Cochrane Handbook for Systematic Reviews of Interventions version 6.1 (updated September 2020). Cochrane, 2020. Available from www.training.cochrane.org/handbook.

\section{Schünemann 2020b}

Schünemann HJ, Vist GE, Higgins JPT, Santesso N, Deeks JJ, Glasziou P, et al. Chapter 15: Interpreting results and drawing conclusions. In: Higgins JPT, Thomas J, Chandler J, Cumpston M, Li T, Page MJ, et al, editor(s). Cochrane Handbook for Systematic Reviews of Interventions version 6.1 (updated September 2020). Cochrane, 2020. Available from www.training.cochrane.org/handbook.

\section{Smagula 2016}

Smagula SF, Stone KL, Fabio A, Cauley JA. Risk factors for sleep disturbances in older adults: evidence from prospective studies. Sleep Medicine Reviews 2016;25:21-30.

\section{Smith 2019}

Smith KJ, Victor C. Typologies of loneliness, living alone and social isolation, and their associations with physical and mental health. Ageing and Society 2019;39(8):1709-30.

\section{Spielberger 1983}

Spielberger CD, Gorsuch RL, Lushene R, Vagg PR, Jacobs GA. Manual for the State-Trait Anxiety Inventory. 2nd edition. Palo Alto, CA: Consulting Psychologists Press, 1983. 


\section{Spitzer 2006}

Spitzer RL, Kroenke K, Williams JB, Löwe WB. A brief measure for assessing generalized anxiety disorder: the GAD-7. Archives of Internal Medicine 2006;166(10):1092-7.

\section{Steptoe 2013}

Steptoe A, Shankar A, Demakakos P, Wardle J. Social isolation, loneliness, and all-cause mortality in older men and women. Proceedings of the National Academy of Sciences 2013;110(15):5797-801.

\section{Sterne 2019}

Sterne JAC, Savovic J, Page MJ, Elbers RG, Blencowe NS, Boutron I, et al. RoB 2: A revised tool for assessing risk of bias in randomised trials. BMJ 2019;366:14898.

\section{Thomas 2015}

Thomas J. Insights in loneliness, older people and wellbeing, 2015. backup.ons.gov.uk/wp-content/uploads/ sites/3/2015/10/Insights-into-Loneliness-Older-People-andWell-being-2015.pdf) (accessed prior to 27 October 2020).

\section{Victor 2005}

Victor C, Grenade L, Boldy D. Measuring loneliness in later life: a comparison of differing measures. Reviews in Clinical Gerontology 2005;15(1):63-70.

\section{Victor 2012}

Victor CR, Yang K. The prevalence of loneliness among adults: a case study of the United Kingdom. Journal of Psychology 2012;146(1-2):85-104

\section{Wang 2017}

Wang J, Lloyd-Evans B, Giacco D, Forsyth R, Nebo C, Mann F, et al. Social isolation in mental health: a conceptual and methodological review. Social Psychiatry and Psychiatric Epidemiology 2017;52(12):1451-61.

\section{Wang 2020}

Wang J, Lloyd-Evans B, Marston L, Mann F, Ma R, Johnson S. Loneliness as a predictor of outcomes in mental disorders among people who have experienced a mental health crisis: a 4month prospective study. BMC Psychiatry 2020;20(1):249.

\section{Ware 1992}

Ware JE, Sherboume CD. The MOS 36-item short-form health survey (SF-36). I. Conceptual framework and item selection. Medical Care 1992;30(6):473-83.

\section{WHO 2001}

World Health Organization (WHO). Men, ageing and health: achieving health across the life span. Available at www.who.int/ ageing/publications/men (accessed 23 October 2020).

\section{WHO 2014}

World Health Organization (WHO). Recognizing adolescence. Available at apps.who.int/adolescent/second-decade/section2/ page1/recognizing-adolescence.html (accessed 10 June 2020).

\section{WHOQOL Group 1998}

The WHOQOL Group. Development of the World Health Organization WHOQOL-BREF quality of life assessment. Psychological Medicine 1998;28(3):551-8. [DOI: 10.1017/ s0033291798006667]

\section{Wilson 2007}

Wilson RS, Krueger KR, Arnold SE, Schneider JA, Kelly JF, Barnes LiL, et al. Loneliness and risk of Alzheimer disease. Archives of General Psychiatry 2007;64(2):234-40.

\section{Yan 2014}

Yan Z, Yang X, Wang L, Zhao Y, Yu L. Social change and birth cohort increase in loneliness among Chinese older adults: a cross-temporal meta-analysis, 1995-2011. International Psychogeriatrics 2014;26(11):1773-81.

\section{AP P E N DICES}

\section{Appendix 1. Cochrane Specialized Register}

\section{Cochrane Common Mental Disorders Controlled Trials Register (CCMDCTR)}

The Cochrane Common Mental Disorders Group (CCMD) maintains an archived controlled trials register known as the CCMDCTR. This specialized register contains over 40,000 reference records (reports of RCTs) for anxiety disorders, depression, bipolar disorder, eating disorders, self-harm, and other mental disorders within the scope of this Group. The CCMDCTR is a partially studies-based register, with more than $50 \%$ of reference records tagged to around 12,500 individually PICO-coded study records. Reports of studies in the register were collated from (weekly) generic searches of key bibliographic databases to June 2016, which included: MEDLINE (1950 onwards), Embase (1974 onwards), PsycINFO (1967 onwards), quarterly searches of the Cochrane Central Register of Controlled Trials (CENTRAL), and reviewspecific searches of additional databases. Reports of studies were also sourced from international trials registries, drug companies, the handsearching of key journals, conference proceedings and other (non-Cochrane) systematic reviews and meta-analyses. Details of CCMD's core search strategies (used to identify RCTs) are on the Group's website, with an example of the core MEDLINE search displayed below.

[MeSH Headings]: eating disorders/ or anorexia nervosa/ or binge-eating disorder/ or bulimia nervosa/ or female athlete triad syndrome/ or pica/ or hyperphagia/ or bulimia/ or self-injurious behavior/ or self mutilation/ or suicide/ or suicidal ideation/ or suicide, attempted/ or mood disorders/ or affective disorders, psychotic/ or bipolar disorder/ or cyclothymic disorder/or depressive disorder/ or depression, postpartum/ or depressive disorder, major/ or depressive disorder, treatment-resistant/ or dysthymic disorder/ or seasonal affective disorder/ or neurotic disorders/ or depression/ or adjustment disorders/ or exp antidepressive agents/ or anxiety disorders/ or agoraphobia/ or neurocirculatory asthenia/ or obsessive-compulsive disorder/ or obsessive hoarding/ or panic disorder/ or phobic disorders/ or stress disorders, traumatic/ or combat disorders/ or stress disorders, post-traumatic/ or stress disorders, traumatic, acute/ or anxiety/ or anxiety, castration/ or koro/ or anxiety, separation/ or panic/ or exp anti-anxiety agents/or somatoform disorders/ or body 
dysmorphic disorders/ or conversion disorder/ or hypochondriasis/ or neurasthenia/ or hysteria/ or munchausen syndrome by proxy/ or munchausen syndrome/ or fatigue syndrome, chronic/ or obsessive behavior/ or compulsive behavior/ or behavior, addictive/ or impulse control disorders/ or firesetting behavior/ or gambling/ or trichotillomania/ or stress, psychological/ or burnout, professional/ or sexual dysfunctions, psychological/ or vaginismus/ or Anhedonia/ or Affective Symptoms/ or *Mental Disorders/ OR [Title/ Author Keywords]: (eating disorder ${ }^{\star}$ or anorexia nervosa or bulimi* or binge eat ${ }^{\star}$ or (self adj (injur ${ }^{\star}$ or mutilat $^{\star}$ )) or suicide ${ }^{\star}$ or suicidal or parasuicid ${ }^{\star}$ or mood disorder ${ }^{\star}$ or affective disorder ${ }^{\star}$ or bipolar i or bipolar ii or (bipolar and (affective or disorder ${ }^{\star}$ )) or mania or manic or cyclothymic ${ }^{\star}$ or depression or depressive or dysthymi ${ }^{\star}$ or neurotic or neurosis or adjustment disorder ${ }^{\star}$ or antidepress* or anxiety disorder ${ }^{\star}$ or agoraphobia or obsess* or compulsi $^{\star}$ or panic or phobi* or ptsd or posttrauma* or post trauma* or combat or somatoform or somati\#ation or medical ${ }^{\star}$ unexplained or body dysmorphi* or conversion disorder or hypochondria* or neurastheni* or hysteria or munchausen or chronic fatigue* or gambling or trichotillomania or vaginismus or anhedoni* or affective symptoms or mental disorder* or mental health).tw,kf. AND [RCT filter]: (controlled clinical trial.pt. or randomised controlled trial.pt. or (randomi\#ed or randomi\#ation).ab,ti. or randomly.ab. or (random* adj3 (administ* or allocat ${ }^{\star}$ or assign ${ }^{\star}$ or class ${ }^{\star}$ or control* or determine $^{\star}$ or divide or distribut $^{\star}$ or expose* or fashion or number or place $^{\star}$ or recruit ${ }^{\star}$ or subsitut ${ }^{\star}$ or treat*)).ab. or placebo*.ab,ti. or drug therapy.fs. or trial.ab,ti. or groups.ab. or (control* adj3 (trial* or study or studies)).ab,ti. or ((singl ${ }^{\star}$ or doubl* or tripl ${ }^{\star}$ or trebl*) adj3 (blind ${ }^{\star}$ or mask ${ }^{\star}$ or dummy*)).mp. or clinical trial, phase ii/ or clinical trial, phase iii/ or clinical trial, phase iv/ or randomised controlled trial/ or pragmatic clinical trial/ or (quasi adj (experimental or random)).ti,ab. or

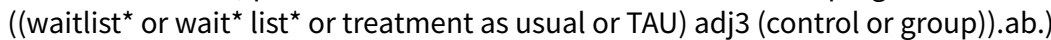

Records were screened for reports of RCTs within the scope of the Cochrane Common Mental Disorders Group. Secondary reports of RCTs were tagged to the appropriate study record.

The rationale of maintaining a comprehensive specialised register was reviewed when the editorial group moved from the University of Bristol to the University of York in June 2016. At this time, the Group decided to archive the CCMDCTR and return to searching the medical and psychological literature directly, on a review-by-review basis.

For this review, the information specialist with CCMD will initially cross-search the CCMDCTR-Studies and References register (all years to June 2016) using the following terms:

\#1 ("social isolation" or loneliness or abandonment or alienation or "separation reactions" or (separation adj1 anxiety) or homesickness or quarantine or lockdown or "lock down" ):eh,emt,ky,mc,mh

\#2 (lonely or loneliness or ((social* or societal $\left.^{\star}\right)$ adj (confine* or isolat $^{\star}$ or inclusion or participation))):ti,ab

\#3 "sense of belonging":ti,ab

\#4 (homesick* or "home sick*"):ti,ab

\#5 (anxiety adj1 separation):ti,ab

\#6 (quarantine ${ }^{\star}$ or lockdown* or "lock down*"):ti,ab

\#7 (( social $^{\star}$ adj3 (contact ${ }^{\star}$ or connect ${ }^{\star}$ or integrat ${ }^{\star}$ or isolat ${ }^{\star}$ or network ${ }^{\star}$ or provision ${ }^{\star}$ or relationship ${ }^{\star}$ or support ${ }^{\star}$ ) adj3 (scale* or subscale $^{\star}$

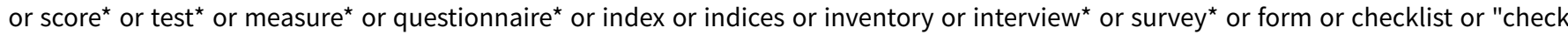
list*")) or ("mental health continuum" adj2 (SF or "short form")) or MHC-SF) [all fields]

\#8 (\#1 or \#2 or \#3 or \#4 or \#5 or \#6 or \#7)

Key to search fields.

ab: abstract; eh,emt: EMTREE headings; ky: Cochrane Review Group (CRG) keywords; mc: MeSH checkwords; mh: MeSH headings; ti:title.

\section{Appendix 2. PsycINFO Ovid search}

Further searches will be conducted directly on the main bibliographic databases, with an example of the PsycINFO search displayed below.

Ovid APA Psyclnfo database $<1806$ to current $>$

Search Strategy:

1. social isolation/ or loneliness/ or abandonment/ or alienation/

2. (loneliness or ((social ${ }^{\star}$ or societal $\left.{ }^{\star}\right)$ adj (confine* or isolat $^{\star}$ or inclusion or participation))).ti,ab,id.

3. "sense of belonging".ti,ab,id.

4. homesickness/

5. $\left(\right.$ homesick $^{\star}$ or home sick ${ }^{\star}$ ).ti,ab,id.

6. separation reactions/ or exp separation anxiety/

7. (anxiety adj1 separation).ti,ab,id.

8. (quarantine? or lockdown? or lock down?).ti,ab,id.

9. or/1-8

10. (loneliness scale? or social network scale? or social provision scale?).af.

11. (loneliness or lonely or (social ${ }^{\star}$ adj (contact? or connect* or isolat $^{\star}$ or network?))).tm.

12. (((mental health continuum) adj2 (short form or SF)) or MHC-SF).af.

13. or $/ 10-12$

Cognitive or behavioural interventions (or both) to prevent or mitigate loneliness in adolescents, adults, and older adults (Protocol)

Copyright (c) 2020 The Cochrane Collaboration. Published by John Wiley \& Sons, Ltd. 
14. cognitive behavior therapy/ or cognitive restructuring/ or cognitive techniques/ or cognitive therapy/ or dialectical behavior therapy/ or schema therapy/ or problem solving/

15. psychotherapy/ or exp group psychotherapy/ or counseling/ or group counseling/ or exp psychotherapeutic counseling/ or psychotherapeutic techniques/

16. mindfulness-based interventions/ or mindfulness/ or "acceptance and commitment therapy"/

17. relaxation therapy/or progressive relaxation therapy/ or autogenic training/ or guided imagery/ or meditation/

18. ((cogniti* or behavio*) adj3 (counsel ${ }^{\star}$ or intervention or therap ${ }^{\star}$ or psychotherap ${ }^{\star}$ or training or treatment or technique* or restructur ${ }^{\star}$ or defusion)).ti,ab,id.

19. (CBT* or CBASP or contingency management or covert conditioning or covert sensititation or defusion or $\mathrm{MBCT}^{\star}$ or problem focus ${ }^{\star}$ or rational emotive or REBT or schema).ti,ab,id.

20. ((problem ${ }^{\star}$ adj2 (focus ${ }^{\star}$ or sol $\left.\left.{ }^{\star}\right)\right)$ or role play ${ }^{\star}$ or schema* or self-control $^{\star}$ or self control $\left.{ }^{\star}\right)$.ti,ab,id,hw.

21. (((psychotherap ${ }^{\star}$ or therap $\left.{ }^{\star}\right)$ adj3 (commitment or acceptance)) or (self* adj (control or analysis or direct* or esteem or help or instruct ${ }^{\star}$ or manage $\left.\left.e^{\star}\right)\right) \cdot$.ti,ab,id

22. ((attribution* or reattribution $\left.{ }^{\star}\right)$ adj3 (therap* or psychotherap $\left.\left.{ }^{\star}\right)\right)$.ti,ab,id.

23. (metacognitive or meta-cognitive or mindfulness ${ }^{\star}$ or third wave or (behavio* adj3 modification) or (thought ${ }^{\star}$ adj3 suppress ${ }^{\star}$ ) or rumination).ti,ab,id.

24. (exposure therapy or exposure task* or psychoeducat* or psycho-educat* or relaxation or sensitivity training or assertiveness training or self talk or (social adj2 (coach* or skill* or effectiveness))).ti,ab,id,hw.

25. exp behavior therapy/ or exp behavior modification/ or behavior change/ or behavior contracting/

26. behavioral activation system/

27. ((behavio* adj1 activ*) or BATD).ti,ab,id.

28. (behavio* adj3 (reinforce ${ }^{\star}$ or re-inforce $\left.{ }^{\star}\right)$ ).ti,ab,id.

29. (behavio* adj2 (change or contracting or modification or modify*)).ti,ab,id.

30. ((activit ${ }^{\star}$ or event?) adj2 (schedul* or training)).ti,ab,id.

31. planned behavior/

32. ((pleas* or enjoyable or rewarding) adj (activit* or event?)).ti,ab,id.

33. exp operant conditioning/

34. (operant conditioning or instrumental learning).ti,ab,id.

35. exp contingency management/

36. (positive interaction* or avoidant coping or environmental contingenc ${ }^{\star}$ or contigency management).ti,ab,id.

37. functional analysis.ti,ab,id,sh.

38. (behavio* and (self adj (care or efficacy or evaluat* or monitor $\left.{ }^{\star}\right)$ )).ti,id,hw.

39. (behavio* adj (counsel ${ }^{\star}$ or intervention or train ${ }^{\star}$ or treatment? or therap ${ }^{\star}$ or psychotherap $\left.{ }^{\star}\right)$ ).ti.

40. (((contingent or positive) adj1 reinforc $\left.{ }^{\star}\right)$ or (reinforc ${ }^{\star}$ adj3 (environment* or experience $\left.\left.^{\star}\right)\right)$ ).ti,ab,id.

41. intervention/ or group intervention/ or group discussion/

42. (group adj2 (program or rehabilitation)).ti,ab,id.

43. or/14-42

44. clinical trials.sh.

45. (randomi\#ed or randomi\#ation or randomi\#ing).ti,ab,id.

46. (RCT or at random or (random* adj3 (administ* or allocat ${ }^{\star}$ or assign ${ }^{\star}$ or class ${ }^{\star}$ or control ${ }^{\star}$ or crossover or cross-over or determine or $^{\star}$ divide $^{\star}$ or division or distribut ${ }^{\star}$ or expose ${ }^{\star}$ or fashion or number ${ }^{\star}$ or place or recruit $^{\star}$ or split or subsitut ${ }^{\star}$ or treat $\left.\left.{ }^{\star}\right)\right)$ ).ti,ab,id.

47. (control ${ }^{\star}$ and (trial or study or group) and (placebo or waitlist* or wait* list* or ((treatment or care) adj2 usual))).ti,ab,id,hw.

48. ((single or double or triple or treble) adj2 (blind* or mask $^{\star}$ or dummy)).ti,ab,id.

49. trial.ti.

50. placebo.ti,ab,id,hw.

51. treatment outcome.md.

52. treatment effectiveness evaluation.sh.

53. mental health program evaluation.sh.

54. or/44-53

55. (9 and 43 and 54)

56. (13 and 43 and 54$)$

57. $(55$ or 56$)$

Key to search fields.

af: all fields; ab: abstract; id: key concepts; hw: subject heading word; md: methodology; sh: subject heading; ti: title, tm: tests \& measures.

\section{WHAT'S NEW}




\begin{tabular}{lll}
\hline Date & Event & Description \\
\hline 18 November 2020 & Amended & Protocol published November 2020, author affiliations updated. \\
\hline
\end{tabular}

\section{HISTORY}

Protocol first published: Issue 11, 2020

\section{CONTRIBUTIONS OF AUTHORS}

$\mathrm{PH}$ and EE: overall responsibility for the protocol and protocol writing, revised the protocol in line with feedback DMcM: oversaw development of the protocol, developed the selection criteria and the methodology

SD: search strategy design

$\mathrm{DE}$ : contributed to the development of the protocol

$\mathrm{NH}$ : contributed to the development of the protocol

EL: contributed to the development of the protocol

RS: contributed to the development of the protocol

NM: contributed to the development of the protocol methodology

SG: developed the concept for the review, and oversaw the writing of the protocol, development of selection criteria, and the methodology

\section{DECLARATIONS OF INTEREST}

$\mathrm{PH}$ : no conflicts of interest

EE: no conflicts of interest

SD: no conflicts of interest

DE: Chief Investigator on studies relevant to the review; will not extract data from his own study or studies if included in the review; no other conflicts of interest

$\mathrm{NH}$ : no conflicts of interest

EL: no conflicts of interest

DMCM: no conflicts of interest

RS: has previously given a webinar on CBT for loneliness, but does not consider this presents a conflict of interest

NM: no conflicts of interest

SG: no conflicts of interest

\section{SOURCES OF SUPPORT}

\section{Internal sources}

- University of York, UK

\section{External sources}

- National Institute for Health Research (NIHR), UK

SG, PH, DMcM time on this protocol was funded by the NIHR Collaboration for Leadership in Applied Health Research and Care Yorkshire and Humber (NIHR CLAHRC YH).

This review is also part funded by the NIHR Policy Research Programme (NIHR200702). 